\title{
Meta
}

Journal des traducteurs

Translators' Journal

\section{PYM, Anthony (1993) : Epistemological Problems in Translation and its Teaching. A Seminar fot Thinking Students, Edicions Caminade, Calaceit (Espagne), 160 p.}

\section{Yves Gambier}

Volume 40, numéro 1, mars 1995

URI : https://id.erudit.org/iderudit/002793ar

DOI : https://doi.org/10.7202/002793ar

Aller au sommaire du numéro

Éditeur(s)

Les Presses de l'Université de Montréal

ISSN

0026-0452 (imprimé)

1492-1421 (numérique)

Découvrir la revue

Citer ce compte rendu

Gambier, Y. (1995). Compte rendu de [PYM, Anthony (1993) : Epistemological Problems in Translation and its Teaching. A Seminar fot Thinking Students, Edicions Caminade, Calaceit (Espagne), 160 p.] Meta, 40(1), 139-143.

https://doi.org/10.7202/002793ar d'utilisation que vous pouvez consulter en ligne.

https://apropos.erudit.org/fr/usagers/politique-dutilisation/ 
- PYM, Anthony (1993): Epistemological Problems in Translation and its Teaching. A Seminar for Thinking Students, Edicions Caminade, Calaceit (Espagne), 160 p.

Au printemps 1992. A. Pym a donné devant ses étudiants de $2^{\mathrm{e}}$ et $3^{\mathrm{e}}$ années de Las Palmas un séminaire de 16 heures sur les problèmes épistémologiques en traduction 
et en interculturel. De la première moitié de ses interventions résulte cet ouvrage. facile à lire, au ton personnel et généreux dans sa visée (partager certaines interrogations touchant à la fois la démarche traductionnelle et la démarche pédagogique). Ne pouvant ni le résumer ni en faire le tour, je tenterai d'en indiquer quelques thèmes et arguments ainsi que quelques limitations, dues aux conditions de production de l'ensemble et au contexte de son énonciation.

Quelles sont les positions principales de l'auteur'? II les rassemble lui-même en partie dans une brève postface (pp. 147-150), non sans certaines distorsions d'optique puisque par exemple la distinction entre les deux types de savoir: interne (ou expertise même du traducteur) et externe (ou crédibilité du traducteur sur le marché des discours). n'est guère explicitée en tant que telle - sauf p. 130 - dans les chapitres antérieurs. Certes les réflexions sur l' "autorité" à gagner vis-à-vis des clients, donneurs d'ouvrage. lecteurs (ch. 7), sur la négociation des erreurs et des fautes (ch. 8)... conduisent graduellement à cette distinction: les doutes liés à l'infinité (théorique) des sens possibless se résorbent et se résolvent dans la finalité sociale du travail commandé. Cette dualité dynamique et non dichotomique permet de dépasser la tentation de l'indécision quand la traduction piétine - p. ex. quand il faut rendre des termes culturels, les imbroglios touchant le concept d’équivalence. l'indétermination sémantique. l'ouverture sans fin de la "sémiosis» (un signe en appelle un autre qui en appelle un autre. elc. I. les frustrations de l'enseignant qui ne peut que sonder le puits sans fond de la "vérité"... l.es interprétations quasi illimitées d'un texte, les disputes aussi quasi illimitées pour tel ou tel rendu, les décisions d'autorité pour telle ou telle correspondance ne regardent ni le traducteur ni le traductologue.

La problématique de la sémiosis (pp. 36-37) peut alimenter les réflexions du poststructuralisme et de la déconstruction (ch. 2), le modèlé de Quine (ch. 3) et d'autres spéculations sur l'univocité parfaite, sur l'absolu en communication.... il n'en reste pas moins que tout texte, «infini potentiel», fonctionne "dans un fini actuel» (pour reprendre des termes de P. Valéry): le sens n'est pas toujours flottant, à la dérive. car il est "pris» dans un contexte pragmatique, énoncé pour certaines raisons et finalités, raccroché souvent à des pratiques culturelles décisives, contraint enfin parfois par des options éthiques/ idéologiques. Le concept ici de retraduction aurat pu trouver sa place autrement que par vagues allusions (pp. 51. 81. 115). On peut sans doute discuter longtemps, dans l'abstraction. de telle ou telle possibilité: ce luxe interprétatif, cette luxuriance gratuite ne résistent guère au fait que le traducteur ne doit proposer qu une solution (relative). toujours ou presque à courte échéance sinon dans la précipitation. On rejoint ici une question centrale déjà posée dans les travaux sur l'intertextualité. à la suite de Bakhtine: si tout discours est réaménagement d'un dit antéricur, si tout signifié présuppose un autre signifié, n'y at-ilil pas régression à l'infini. une circularité intenable? Par le système de renvois, de glissement progressif des significations, le sens ne se marque-t-il pas et ne s'efface-t-il pas sans cesse? Oui, en théorie; non, en pratique car on ne peut faire l'économie de l'histoire et des conditions sociales d'énonciation. De même, l'historicité des situations de traduction surdétermine les choix à faire, les sens à faire comprendre: la traduction est une activité intellectuelle à finalité sociale, son obligation de résultat étant son obligation de faire sens, hic "t munc. Le traducteur joue avec cette double dimension: il négocie son délai avec son commanditaire, pour se donner le temps de réfléchir. de décider, tout en sachant que ce commanditaire cherche à atteindre un but, à réaliser un effet par l'intermédiaire du texte traduit.

A. Pym ne s'arrête pas là - ce qui ne serait finalement que la reprise d'oppositions déjà largement ressassées, me semble-t-il. en traductologie jusqu au refrain nauséeux. Ses réflexions, traversées par la systématique du doute (ch. 1), vont plus loin: la déconstruction. l'indéterminisme, quelles qu'en soient les limites au niveau de leurs apports 
philosophiques, permettent de remettre en cause certaines «autorités», jusque-là fondatrices dans quantité de modèles et d'efforts de théorisation en traduction. Ainsi les intentions de l'auteur, la déférence envers l' «original» n'ont pas de raison d'être dans les justifications des choix faits par le traducteur. Si le sens est prospectif. co-construit, sinon même projectif. subjectif - et non caché dans le texte, figé une fois pour toutes, il ne peut s'imposer: les "autorités» traditionnelles - l'auteur, l'enseignant, le dictionnaire (bilingue), les informateurs natifs, etc. - n'ont aucune clé privilégiée ou option supérieure. Elles ne peuvent être la police du sens, ne peuvent commander une traduction-modèle, une solution unique. Il faut reconnaître ici que ces problèmes de l'auteur. plus ou moins fétichisé, du sens plus ou moins sacralisé, encombrent encore nombre d'approches prescriptives en traduction, nombre de manuels de version-thème, nombre de discussions sur la traduction comme moyen d'apprentissage, nombre de propositions aussi dans les orientations traductologiques pragmatiques, sinon fonctionnalistes. Mais Pym ne polémique pas avec ces approches ou orientations: ses interrogations débouchent sur la problématique de l'enseignement de la traduction (ch. 4, 5). Que faire et comment faire en effet si, en classe, on ne peut en rester qu 'à la sémiosis (plaisir de l'interprétation ouverte, non contraint par le principe de réalité). si les outils pédagogiques - y compris l'enseignant - n'ont plus le demier mot?

Le chapitre 6 propose quelques principes pouvant guider la formation:

- l'enseignement doit être inductif (voir aussi pp. 126-128) - on ne part pas de règles, d'un corps théorique: on apprend à traduire en traduisant (voir aussi p. 92), selon une tautologie qui n'est pas sans rappeler l'affirmation qu'on apprendrait à nager en sautant dans l'eau :

on doit discuter non des fautes de langue (de transcodage) mais des erreurs (relatives) de traduction et cela oralement et si possible collectivement - manière d'initier l'apprenant à poser des questions, à motiver ses choix plus tard dans son face à face avec son donneur d'ouvrage :

- l'enseignant doit être un praticien: Pym ne cache pas ici (pp. 105-107) les ambiguîtés générées alors par l'institution, mais par ailleurs il ne s'inquiète pas de savoir si le praticien est nécessairement un pédagogue:

- l'encouragement à la discussion, le rejet des solutions d'autorité, le recours aux échanges d'étudiants, sinon aux classes mixtes (natifs / non natifs)... conduisent à traiter les apprenants comme des adultes - contre des rapports hiérarchisés de manière rigide, contre une didactique maternante, parfois infantilisante ( $f$. pp. 54-55):

- la recherche d'équivalence (consensuelle) est la fin de l'effort traductionnel: elle est à la fois sa visée et met fin à l'exercice en cours.

Peut-on dire que les thèses de Pym sont simples ou radicales, nouvelles ou déjà partagées? Cela dépend bien sûr des conceptions auxquelles on se réfêre, des situations d'enseignement auxquelles on pense.

Sans prétendre au point de vue de Sirius, au statut de vigie, j'émettrai quelques remarques et critiques - moins d'ailleurs sur les positions et propositions de l'auteur que sur sa manière de les présenter, sur sa démarche. Il nous dit dans sá préface que l'ensemble a élé esquissé pour des étudiants engagés dans la tradition et le contexte canarien-espagnol: c'est vrai que le ton semble parfois à usage inteme (pp. 26, 105-113, 128-129..). Cela justifie-t-il malgré tout le plaidoyer pro domo confinant à l'autobiographie (pp. 40, 44-46...) ou confondu avec l'anecdotique (pp. 45, 8.5, 1(14...). l'auto-justification embrouillée dans une vague polémique (pp. $43,55,84 \ldots$ )? Après une cinquantaine de pages, on peut se demander encore ce qu'il faut entendre par "problèmes épistémologiques», "enseignement de la traduction»... Cela nécessite-l-il de toute façon d'apostropher un auditoire «non pensant " ou insuffisamment pensant (cf. titre du livre: pp. 11,110 ) - paradoxe qui 
confine à la contradiction avec une des assertions finales (p. 108) qu'il faut voir les apprenants comme des «adultes», déjouant ainsi tout rapport de condescendance ?

Ce malaise devient plus fort quand en contrepoint de la déclaration, justifiée, d'antiautoritarisme, l'auteur multiplic les «je» d'autorité: certes, face à la situation qu'il connaît et qu'il cherche à changer (p. ex. pp. 14-15), A. Pym prend légitimement position. Mais tous ces «je» ne sont pas que tactiques. Sa surdité à certaines questions de son public (pp. 61-62, 76-77, 87, 1(09-111,.) - reconnue avec justesse p. 12 - inaugure mal aussi de la mise en place d'une pédagogie de l'interaction. Peut-être ce malaise n'est-il dû en fait qu'à la transformation de la forme "conférence" (voir pp. 11, 14-15) en celle d'un «essai»? A. Pym souligne lui-même la différence entre les deux codes, oral/écrit (pp. 15, 38): il ne semble pas en avoir tiré toutes les conséquences. L.énoncé devant un auditoire peut vibrer de la présence de l'énonciateur. peut se répéter sans lasser. peut reprendre un point selon une évolution en boucle; cela n implique pas que les mêmes recettes passent dès lors qu'on est à l'écrit. Ainsi. des rapprochements, des renvois auraient facilité la lecture (p. ex. pp. $54-55$ et $108 ; 90-92$ et ch. $7 ; 108$ et ch. 8...), auraient rendu certains fils rouges plus perceptibles... sans forcément tomber dans un texte académique, cartésien, péremptoire. La persuasion aurait gagné avec un travail plus condensé, plus élagué.

Autre réserve: A. Pym cherche, là aussi légitimement, à dépasser les approches philologiques, spéculatives. Mais ne réduit-il pas malgré lui les problèmes et solutions de traduction au seul niveau du mot (p. ex. traités: manzamos pp. 68-70; momte/ hush pp. 71-73 et 83: ganadero/drover pp. 73-76; liste de "réalités" australiennes pp. 93-95; pared/ muralla/wall pp. 117-118)? Ne les réduit-il pas à la seule problématique des termes culturels? Cette réduction expliquerait alors les confusions ou à tout le moins les hésitations entre traduction et transcodage (pp. 89.97...). en dépit des efforts pour lever toute ambiguïté à ce propos (pp. 26-30). Elle expliquerait aussi l'étrange fixation contre Newmark (pp. 29. 43, 67. 80)-81, 149. sans cublier la référence de Pym 1992h), alors que peu d'auteurs en traductologie sont cités (2 pages de bibliographie seulement, sans inclure les travaux de Pym lui-même) quand hien même auraient pu être convoqués p. ex. Meschonnic - p. 56 sur l'échange/change, Ladmiral - p. 58-60) sur la traduction comme dérivée de ce qu'on comprend et de ce qui est acceptable à un moment donné. Pym ne tombe-t-il pas en partie dans le piège qu il dénonce, ne mime-t-il pas (p. 40, à propos de Derrida) l'attitude de rejet autoritaire qu affectionne justement Newmark'? Retour du refoulé ? Il n'est jamais aisé de désapprendre et de transformer un paradigme! Dans cette même perspective, on peut regretter également la prédominance - encore - des exemples littéraires ; certes l'auteur réfère aussi à des expériences pragmatiques (p. 106, ch. 8) mais moins pour fonder son analyse que pour l'illustrer, comme si le non-littéraire n'avait que valeur anecdotique.

Une autre question lentement se pose, au cours de la lecture, qui n'est pas sans conséquence sur les observations concernant l'enseignement de la traduction: A. Pym fait part du silence de ses éludiants, après énoncé à voix haute d'un passage douteux pour lui (pp. 68-69, 93): ailleurs (pp. 88. 90-92, 97. 102...). il mentionne les choix et stratégies que peut suivre le traducteur qui n'est pas une machine à labriquer du mot à mot (p. 29): en scrutant ses exemples ( $p p .68-75,93-95,121-122 \ldots$ ). il met en évidence la complexité des connaissances nécessaires pour décider de l'équivalence appropriée: tout cela n'indique$t$-il pas que la formation ne peut se limiter à la pratique, même raisonnée. de la traduction? Que d'autres savoirs concourent à la capacité d'analyser. de produire, de sélectionner un texte adéquat? Comment dès lors intégrer - sans forcément sacraliser le travail de traduction (p. 90) - ces apports? La question n'est pas triviale, quand on insiste aujourd hui tant sur l'interdisciplinarité à la base de la maîtrise du traducteur, à la source de son savoir-faire, de ses compétences ( pp. 27-28). Les lacunes ici font douter de la belle assurance de certains principes formulés au chapitre 6 : peut-on discuter ou suggérer des 
alternatives de traduction, sans comprendre ce dont on parle, sans connaître les potentialités des langues et des cultures? Faut-il toujours redécouvrir la lune (problème des traductions des noms propres (p. 104), des métaphores, de l'humour, etc.), comme si les expériences acquises $n$ 'étaient $d$ 'aucune valeur ou étaient intransmissibles ? L'exigence d'«intelligence» (innée?), la non-difficulté du traduire (p. 91) ne reviennent-elles pas à renforcer le préjugé que le bagage des langues suffit pour traduire - le reste étant donné en surcroît, ou pire n'aboutissent-elles pas, derrière une volonté démocratique, consensuelle, à donner un caractère élitiste à une profession pourtant en mal de reconnaissance? Cette ambiguiité ne vient-elle pas justement du statut universitaire de celui qui parle (pp. 105-106)? On a l'impression que la nécessité de former systématiquement les traducteurs s'est vidée de toute substance (doute confirmé p. 129 , p. ex.); en tout cas, on ne voit plus pourquoi par exemple la formation se fait dans l'enseignement supérieur et non dans une école professionnelle.

D'autres questions, convergentes, se posent: elles restent sans réponse. La traduction est-elle si simple et entière? Faut-il commencer par un type de traduction et lequel? Comment en décider? (p. I(10) Avec quels textes travailler et pour quelles finalités? Comment passe-t-on de la pédagogie unilatérale à la pédagogie participante? (p. 103) Peut-on évacuer d'un revers de main la question de l'évaluation, le bachotage ou la peur de certains examens (pp. 129-130) ne se confondant pas avec la critique (p. 87), l'autoévaluation (p. 109) ou la mesure de la progression dans l'acquisition des compétences (pp. 110-111)!

Demière remarque: les exemples du ch. 8 - sur la discussion des fautes et erreurs — sont-ils tous de même nature? Les positions afférentes qu iils représentent sont-elles à mettre sur le même plan'? Certains de ces exemples ne vont pas sans naîveté (exprès?): pp. 13.3-1.34 sur les effets de la méconnaissance de la langue de départ (à propos de député-maire, de mon petir (hou); d'aucuns, bien connus par les praticiens, semblent liés au marché et à des représentations sociales de la traduction (texte de départ défectueux: p. 133 ; donneur d'ouvrage se prétendant plus apte que le traducteur: pp. 140-143; efforts à déployer pour se faire payer: p. 146); d’autres ressortent de la panoplie des stratégies possibles (pp. 135-139: traduction par évitement, par confession de ses lacunes, par proposition d'alternative) - laissant un peu sur notre faim par leur apparente désinvolture; enfin, quelques-uns sont des assertions trop peu démontrées pour emporter l'adhésion (contre l'excessive confiance en la terminologie: p. 143; contre l'hyperspécialisation, tout en reconnaissant la nouvelle division internationale du travail: pp. 144-145).

On le voit. l'ouvrage recensé aborde une multitude de points cruciaux à la fois pour l'essor d'une théorie de la traduction et pour le développement d'une didactique de la traduction. Il le fait souvent avec conviction et clarté. II n'est pas sûr cependant que l'édition actuelle favorise toujours l'exposé. l'argumentation. La forme orale peut aider à avancer pas à pas, à mémoriser : l'écrit ne respire pas de la même manière: il ne compose pas avec le lecteur comme un séminaire avec ses auditeurs. Mais peut-être est-ce là un différend plus culturel qu un désaccord sur le fond.

YVES GAMBIER 